\title{
A Versatile Acoustic Beacon for Navigation and Remote Tracking of Multiple Underwater Vehicles
}

\author{
Nuno Cruz, Luís Madureira, Aníbal Matos and Fernando Lobo Pereira \\ Faculdade de Engenharia da Universidade do Porto \\ Instituto de Sistemas e Robótica \\ R. Dr. Roberto Frias, 4200-465 Porto \\ Portugal \\ \{nacruz, lmad, anibal, flp\}@fe.up.pt
}

\begin{abstract}
In this paper, we present a low-cost versatile device developed to support the navigation of autonomous underwater vehicles. Unlike the usual transponders extensively used for LBL acoustic navigation of a single vehicle, this device allows the navigation of multiple vehicles and the remote tracking of their positions, without any extra acoustic signals being transmitted.

The navigation beacon has a radio buoy connected to an underwater reconfigurable multi-frequency transponder. A remote tracking station receives data from the buoy and monitors the position of the vehicles in real time. We describe the mechanical, electronic and software modules involved, as well as the tracking algorithm, and we also present experimental data from an operational mission.
\end{abstract}

\section{INTRODUCTION}

One of the main technological challenges associated with the operation of Autonomous Underwater Vehicles (AUVs) is navigation [1,2]. End-users need data to be gathered along specific trajectories, defined in some local or global reference frame. These trajectories are converted into a path the vehicle has to follow and this, in turn, requires the continuous knowledge of its position. On the other hand, there is always the risk of loosing an AUV while performing autonomous operations. The external tracking of the vehicle allows for a high degree of confidence, as it becomes possible to monitor its behavior far from the operations area. Acoustics play a critical role in most navigation and tracking systems for underwater devices. Since electromagnetic waves hardly propagate in seawater, sound waves are generally used to measure ranges to beacons installed in known positions.

In this paper we present the latest developments in an acoustic navigation system specially designed and implemented for the operation of the Isurus AUV. The motivation for this new device arises from the lack of navigation systems able to cope with multiple vehicles sharing the same acoustic channel. In our case, the reconfigurable multi-frequency transponder replaces a set of traditional transponders in a convenient single packaging. In order to track AUVs, it is common to send extra acoustic signals to the vehicles and detect ranges and/or bearings to determined positions. With our navigation beacon, just by listening to the acoustic signals exchanged between the vehicles and the transponders, it is possible to detect the position of the vehicles in real time. Furthermore, our tracking algorithm works independently of the transponder location, which means that we can even use it in the AUVs. In this way, each vehicle can determine in real time the position of all the others within the same acoustic network without any other means of inter-vehicle communication (such as acoustic modems).

This paper is organized as follows. In section 2 we describe the Isurus AUV and the navigation system in particular. In section 3 we present the new navigation beacon. In section 4 we describe the tracking system. Finally, we present some experimental results achieved in a real scenario.

\section{THE ISURUS AUV}

Autonomous Underwater Vehicles constitute powerful and effective tools for underwater data gathering. These vehicles operate with no physical link with the operator, carrying a set of relevant sensors to characterize the underwater environment. The Systems and Underwater Technology Lab (LSTS) at the University of Porto has been operating and customizing the Isurus AUV, for the past 4 years [3].

Isurus (Fig.1) is a REMUS (Remote Environment Measuring UnitS) class AUV, built by the Woods Hole Oceanographic Institution, MA, USA, in 1997. These vehicles are low cost, lightweight AUVs specially designed for coastal waters monitoring [4]. The reduced weight and dimensions makes them extremely easy to handle, requiring no special equipment for launching and recovery.

Isurus has a diameter of $20 \mathrm{~cm}$ and is about 1.5 meters long, weighting about $35 \mathrm{~kg}$ in air. Inside the hull several subsystems have been improved or specifically developed at LSTS, contributing to the continuous enhancement of the vehicle performance and reliability. The maximum forward speed of the vehicle is 4 knots, however the best energy efficiency is achieved at about 2 knots. At this velocity, the energy provided by a set of rechargeable Lithium-Ion 
batteries may last for over 20 hours (i.e., over 40 nautical miles).

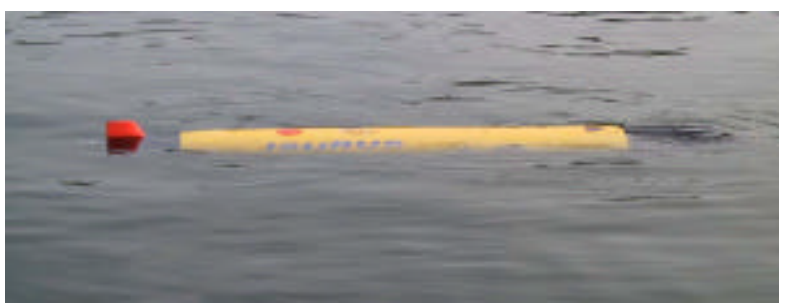

Fig.1 - The Isurus AUV at the surface

Although small in size, this vehicle can accommodate a wide range of oceanographic sensors, such as CTD, Altimeter, Sidescan Sonars, ADCP (Acoustic Doppler Current Profiler), Optical BackScatter, etc.

\section{A. The Isurus Navigation System}

The main function of the navigation system is to determine the position of the vehicle in the horizontal plane, as the vertical coordinate of the vehicle is given directly from the pressure cell.

The vehicle carries an omni-directional transducer, capable of transmitting and receiving acoustic signals. The vehicle interrogates each beacon, or transponder, with a unique frequency and each transponder replies with another frequency. The navigation system measures the turn around time and computes the distance to that transponder. Using a triangulation algorithm and knowing the locations of the beacons, the vehicle position can then be determined (this process is usually known as long baseline navigation, or LBL).

Since the vehicle can be a few kilometers away from each transponder, there might be a few seconds between position estimates. In the meantime, the navigation system uses dead reckoning information to update the position estimate. The instantaneous velocity is obtained by measuring the propeller rotation speed and the vehicle heading, pitch and roll. Velocity measurements are fused together with range measurements by a Kalman filter based algorithm [5], taking advantage of the characteristics of each type of data. On one hand, the vehicle velocity is available at a high rate, but its integration leads to a drift in the estimated position. On the other, range measurements, available at a lower rate, can be noisy but do not drift over time. The algorithm updates the estimate of the vehicle position at the same rate the velocity is measured, and corrects it whenever a new range measurement is available, giving the best estimated position in real-time [6]. Besides the estimation of the vehicle position, the algorithm also produces a coarse estimate of the water current (in the horizontal plane).

\section{THE NEW NAVIGATION BEACONS}

The acoustic navigation beacons were developed to allow for standard LBL navigation and for the remote tracking of multiple underwater vehicles. A typical configuration of such a device is sketched in Fig. 2. It consists on a surface buoy connected to an underwater multi-frequency acoustic transponder in the $20-30 \mathrm{KHz}$ frequency range.

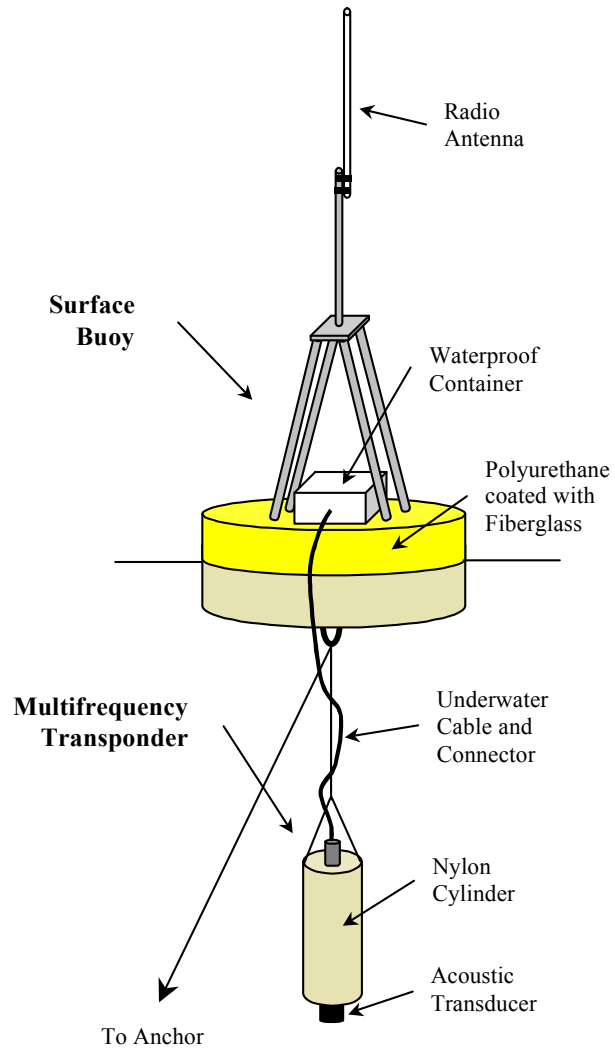

Fig. 2 - Navigation beacon

The multi-frequency transponder substitutes the functionality of a set of conventional transponders in a single location, i.e., it has a pair of frequency pairs, so that when a given frequency that is detected, the corresponding frequency is transmitted. A network of these transponders, with no surface buoys, is sufficient for the navigation of multiple AUVs, since each vehicle uses a unique pair of frequencies for the same transponder location. However, it does not allow for remote tracking of their positions.

In order to track the position of a vehicle, the transponder has to detect that the vehicle is sending an acoustic signal to another transponder. It does not reply to these signals, but it relays this information to shore, where it is processed to resolve the vehicle's position, according to a simple algorithm described later. To transmit this information, the transponder needs to be connected to a surface buoy, where the radio modem and antenna are installed. 


\section{A. Multi-frequency Transponder}

The fundamental building block of the navigation beacon is the multi-frequency transponder. The electronics for the transponder are similar to the electronics used for the AUV navigation system. They consist of three basic modules, namely a multi-channel acoustic receiver, a multichannel acoustic transmitter and a processor unit.

The multi-channel acoustic receiver is directly connected to the acoustic transducer, operating in the 20$30 \mathrm{Khz}$ frequency range. The incoming acoustic signals are pure tones and the receiver has a set of analog filters with high $\mathrm{Q}$ factors, each one tuned to a different frequency. The output of each of these filters is connected to an envelope and level detector circuitry. A digital detection signal is generated whenever the output level of an envelope exceeds an adjustable threshold. These thresholds can be set to take into account the environmental acoustic noise and the expected ranges. The current version has 8 filters in parallel, so it is capable of detecting 8 different acoustic signals simultaneously. This number may be increased if required, by adding the corresponding number of filters.

The multi-channel acoustic transmitter is connected to the same transducer as the receiver. It is able to send an acoustic signal, digitally stored in memory. Currently, we can choose from 8 pure tones, each one lasting up to $16 \mathrm{~ms}$.

Finally, the processor unit is a micro-controller board that monitors the incoming signals, computes the expected delay and replies with the proper signal, according to a predetermined set of $\mathrm{Rx} / \mathrm{Tx}$ pairs. This board is based on a DS87C520 micro-controllers, from Dallas Semiconductors, that keeps an internal clock with a resolution of $250 \mu$ s. Whenever an acoustic signal is detected, it time-stamps the event and sends an appropriate message through the radio modem. This radio link also allows for the remote reconfiguration of the frequency pairs (stored in memory) without recovering the transponders. This feature is particularly useful when the transponders are installed in existing moorings for long periods of time.

The whole system was designed for very low power consumption. A pack of primary cells provides about $100 \mathrm{Wh}$ of energy, which is enough for a few weeks of continuous operation (obviously dependant on the number of signals transmitted). The processor unit is permanently monitoring the voltage level at the batteries and sends a warning signal whenever it drops below a certain value.

All the modules are assembled together with the primary battery pack in a $30 \mathrm{~cm}$ long cylinder weighting about $4 \mathrm{~kg}$ in air (Fig. 3). The dual o-ring nylon cylinder was manufactured in a local machine shop and the total cost of the equipment is about $\$ 1,500$ USD.

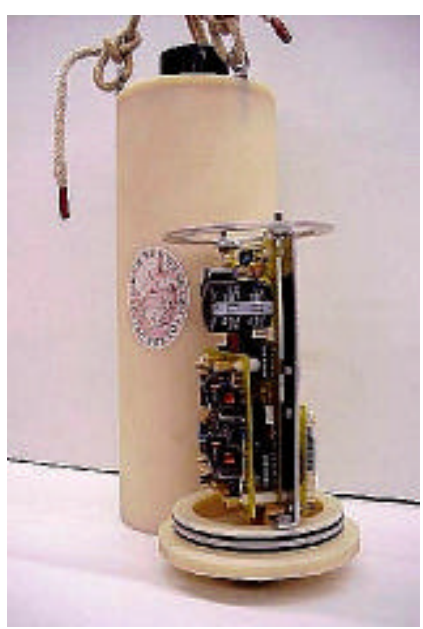

Fig. 3 - Multi-frequency transponder

\section{B. Surface Buoy}

The surface buoy is required to establish communication between the underwater transponder and a remote station. The flotation is obtained from a disk of polyurethane foam coated with a thin layer of fiberglass. For low current environments, this disk has a diameter of $50 \mathrm{~cm}$, resulting in a buoyancy of over 10kgf. This is the version that has been used in the Douro river (Fig. 4). If the mission is to be performed in a harsher environment (with high currents and strong winds), this diameter can easily be increased to achieve greater stability.

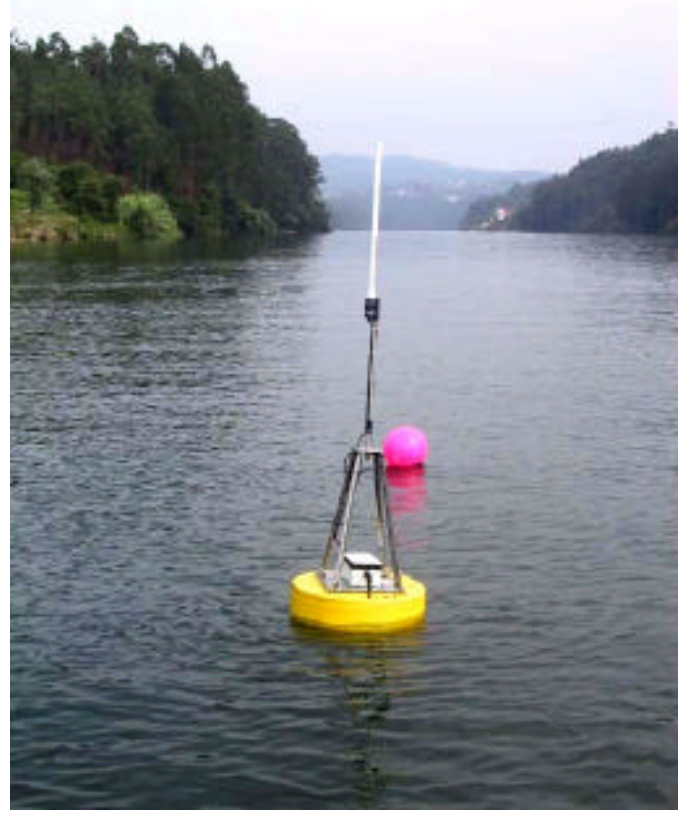

Fig. 4 - Surface buoy in the Douro river

At the top of the buoyant disk, an IP67 watertight container holds a X7200 radio modem, from Warwick Industrial Electronics, Ltd., in the UK. This radio modem 
can transmit data at 19200 baud. The modem is connected to an end-fed dipole antenna, which has unity gain and provides omni-directional radiation. It is installed $1 \mathrm{~m}$ above water level, allowing for a maximum range of $20 \mathrm{~km}$, if a directional antenna is used at the receiving station.

Inside the container, there is also a Garmin GPS25 (an OEM GPS receiver) with a small antenna. This unit outputs Lat/Lon positions once a second, which is enough to detect any significant drift in the buoy and allow for post-mission corrections of navigation data.

An underwater cable establishes the serial connections between the micro-controller at the transponder and the radio and GPS at the surface. In the current configuration, this cable also provides power to the waterproof container. Later on, power will be provided by rechargeable batteries and solar panels located in the buoy frame.

Both the mechanical frame and the flotation disk were also manufactured in a local machine shop, weighting about $15 \mathrm{~kg}$. The total cost for the surface buoy, including the electronics, was kept below \$2,000 USD.

\section{Operational Setup}

For typical LBL navigation algorithms, such as the one described earlier, each AUV needs to be within acoustic range of at least 2 acoustic transponders (providing the AUV never crosses the baseline). In order to be able to track the AUVs in real time, one of these transponders needs to be connected to the surface buoy and radio transmit data to the shore station. To exemplify this, consider the case of a $8 \mathrm{~km} * 4 \mathrm{~km}$ area of operation. For cost minimization, the baseline should be constructed with 5 multi-frequency transponders, but only 2 of them need to have surface buoys (Fig. 5).

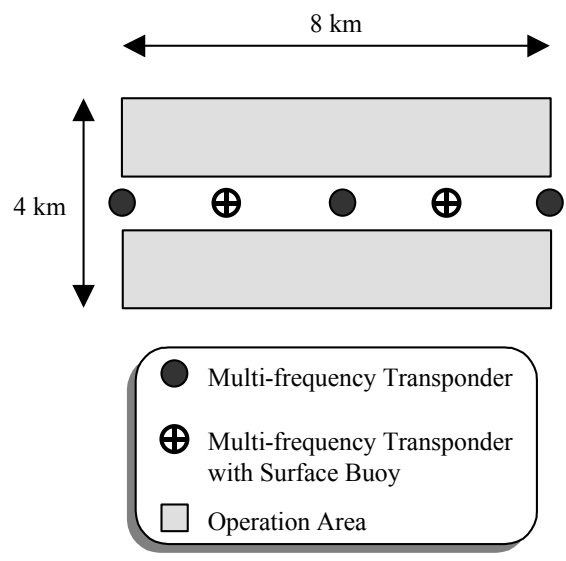

Fig. 5 - Navigation Beacons for an operation area

With this arrangement, multiple AUVs can navigate in both sides of the baseline at the same time, providing they stay within one of the shaded areas. At the same time, they can be tracked from a remote station, since they are always within acoustic range of one of the beacons with radio. The size of the gap between the two shaded areas arises from the increase in the position errors as the vehicles get close to the baseline. As a rule of thumb, the AUVs should not get closer to the baseline than around $1 / 4$ of the distance between consecutive transponders [6]. This results in an effective operational area of $8 \mathrm{~km} * 3 \mathrm{~km}$. Note that the frequencies that we use are intended for relatively small areas (the detection range is about $3 \mathrm{~km}$ ). For larger operation areas, the acoustic signals need to have lower frequencies.

\section{EXTERNAL TRACKING STATION}

\section{A. Tracking Algorithm}

To illustrate the tracking mechanism, lets assume that AUV\#1 is using transponders A and B to navigate. Under normal operation, it will transmit frequency $\mathrm{fl}$ to transponder A and wait for the reply signal. As soon as this reply is detected, it will transmit frequency $\mathrm{f} 2$ to transponder B and wait for the reply from transponder B. This sequence will proceed during the mission execution. Any listening device tapping the acoustic channel will detect all transmissions. The time elapsed between the detection of frequency $\mathrm{fl}$ and the detection of $\mathrm{f} 2$ corresponds to twice the range between the vehicle and transponder $\mathrm{A}$; the time elapsed between the detection of frequency $\mathrm{f} 2$ and the detection of frequency $\mathrm{fl}$ corresponds to twice the range between the vehicle and transponder $\mathrm{B}$. The same algorithm applies for other AUVs using different frequencies.

Providing that the listening device is within acoustic range to the vehicles, this mechanism works independently of the location, as it only deals with time differences. In fact, we have tested it in the past with PAL (for Portable Acoustic Locator), a small listening device that was connected to a laptop computer and was carried in a small boat during operations. With the new navigation beacon, the listening device is now part of the multi-frequency transponder.

The multi-frequency transponders have analog filters for all frequencies used by the vehicles, and the radio modems transmit a time-stamped message whenever each vehicle interrogates each transponder. Since the vehicles are consecutively switching from one transponder to another, we can measure the time between interrogations and, so, determine the range to each of the transponders. On a shore station or in a small boat, we only need to receive the messages transmitted by the radio modems in order to track the vehicles. It should be stressed that this process is only possible with the new vehicle software [7], implemented on the QNX real time operating system. This software ensures that each vehicle switches to the other transponder immediately after detecting a response (the uncertainty in this switching time is kept below $1 \mathrm{~ms}$ ). 


\section{B. Emergency operation}

The above behavior requires a normal operation of the on-board software. Should a failure occur, a tracking mechanism becomes even more valuable.

The navigation system of the AUVs possesses an automatic acoustic responder that replies with a specific signal whenever an emergency interrogation is received. This system has a backup battery that keeps it working in the event of a failure in the main power supply. From the shore station, it is then possible to send a command for the multi-frequency transponder to interrogate the vehicle in this emergency channel, and wait for its reply. Measuring the elapsed time, the distance between the transponder and the vehicle is computed. The vehicles' positions can then be determined by simple triangulation.

A different emergency scenario occurs whenever there is the urge to abort the mission before completion. For this purpose, there is a software watchdog running on the vehicle and if a series of signals are detected in a short period of time, typically 5 detections in 3 seconds, the running mission is aborted.

\section{Tracking Software}

The tracking software runs in a computer connected to a radio modem that receives data from the surface buoys and displays the position of the vehicles in real time (Fig. 6).

The software also displays and logs the GPS position of the tracking buoys. This data is relevant for post-mission analysis of navigation data, since the navigation system of the AUVs assumes that the transponders are stationary during the whole mission.

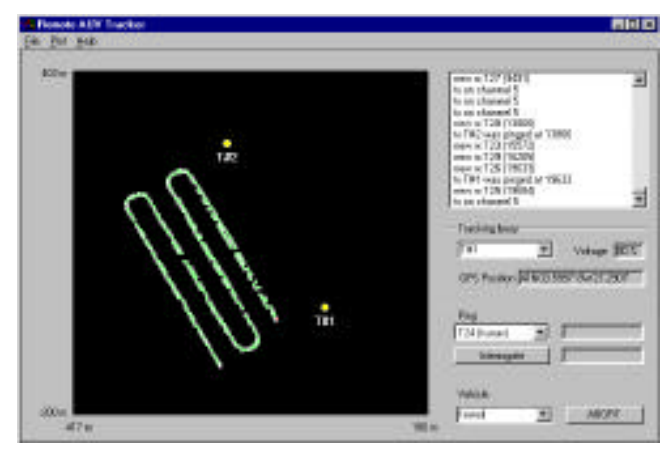

Fig. 6 - Tracking software

In an emergency scenario, it is possible to press the "Abort" button, which causes all the transponders to send acoustic signals in the proper emergency channel. Alternatively, one can use the interface as a front-end to the multi-frequency transponders, i.e., command it to send acoustic signals in any channel, reconfigure the frequency pairs, report battery status, etc.

\section{EXPERIMENTAL DATA}

The navigation system described in this paper has been successfully tested in real application scenarios, such as the Douro river, close to Porto, in Portugal. Fig. 7 shows the trajectory of the Isurus AUV in a simple run. During this experiment, we used one of the new navigation buoys (T\#1) and a conventional transponder ( $\mathrm{T} \# 2)$. The continuous line shows the internal navigation data from the vehicle logging system; the crosses show points at which the external tracking was available. At the moment, there is no filtering in the tracking data, as it is mainly intended for general overview of the behavior of the vehicle.

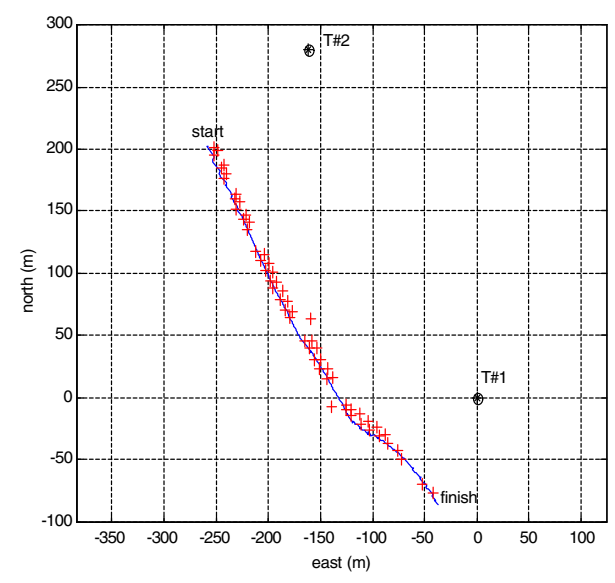

Fig. 7 - Vehicle navigation data vs. external tracking

It should be stressed that the external tracking data can only be updated whenever there is an exchange of acoustic signals between the vehicle and the beacons. Every position update is computed as soon as a new range is available, but it needs to use another range that may be a few seconds "old". This results in a small zig-zag pattern that can be about $5 \mathrm{~m}$ wide.

The GPS position of the surface buoy was also logged during the mission, so that we could quantify the drift from the deployment point (Fig. 8).

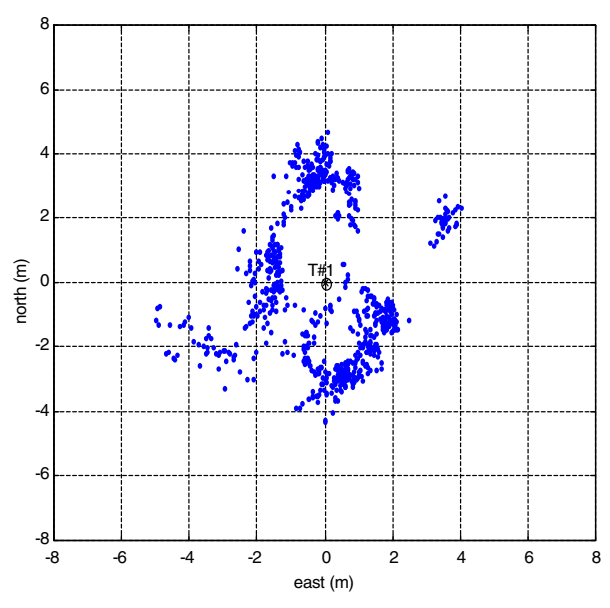

Fig. 8 - GPS data for the navigation beacon 


\section{CONCLUSIONS}

The multi-frequency transponder proved to be an effective solution for the replacement of a set of conventional LBL transponders in a single, convenient packaging. They allow for the navigation of multiple AUVs, and they can easily be reconfigured by software.

The tracking mechanism described in this paper is an efficient solution for the external monitoring of the positions of multiple AUVs in the same acoustic network, as it relies on the proper operation of the navigation system of the vehicles. Typical approaches, some of them commercially available, require the vehicle to communicate its position or to send special signals for the sole purpose of external tracking. Both solutions consume on-board processing time and power, which are valuable resources.

Post mission comparison between internal navigation data and external tracking showed an agreement within 5 to 10 meters. This mismatch arises naturally from the tracking algorithm and it is slightly bigger than the one achievable with commercially available solutions. However, it is perfectly acceptable given the main objective of getting an estimate of the position of the vehicles.

The same mechanism that is used to track the vehicles can be implemented in the vehicles software, so that each AUV can compute, in real time, the position of all the others navigating in the vicinity.

The new navigation beacons constitute a rapid deployable infrastructure for precise navigation of multiple vehicles, as they can be placed with no anchor and the drift information (obtained from GPS) can be acoustically transmitted to the vehicles.

The tracking software provided a convenient tool for monitoring the motion of the AUV during missions. The graphical display is a comprehensive interface to visualize the events that take place underwater.

The navigation beacon presented in this paper is currently in use for the navigation and remote tracking of the Isurus AUV. It has already been successfully tested both during normal operation and under artificial emergency situations.

\section{FUTURE WORK}

The navigation system of the AUVs provides very accurate estimates of the position, relative to the transponder network. However, the transponder location can change during the mission, particularly as we move into deeper waters. We are planning to install a local DGPS reference station, so that the GPS data from the buoys can be corrected and any drift in the position of the buoys can be determined. This will allow for a more accurate postmission correction of the navigation data of the AUVs.
In the current configuration, the thresholds for the detection of acoustic signals need to be set by hardware. In the near future, we intend to implement a digitally controlled circuitry, so that the detection levels may also be set remotely by software.

The surface buoys may have an important role as realtime environment monitoring stations. With the current infrastructure, it will be extremely easy to install underwater and atmospheric sensors and send their data to the shore station.

Past missions have been relatively brief, lasting one to two hours. We are currently preparing for several hours missions off the coast of Aveiro. This will constitute a test for the robustness and effectiveness of the whole navigation system.

\section{REFERENCES}

[1] J. Leonard., A. Bennett, C. Smith and H. Feder, "Autonomous Underwater Vehicle Navigation", MIT Marine Robotics Laboratory Technical Memorandum 98-1, 1998.

[2] H. Singh, J. Catipovic, R. Eastwood, L. Freitag, H. Henriksen, F. Hover, D. Yoerger, J. Bellingham and B. Moran, "An Integrated Approach to Multiple AUV Communication, Navigation and Docking," Proceedings of the MTS/IEEE Oceans'96 Conference, Ft. Lauderdale, FL, USA, 1996.

[3] N. Cruz, A. Matos, A. Martins, J. Silva, D. Santos, D. Boutov, D. Ferreira and F. L. Pereira, "Estuarine Environment Studies with Isurus, a REMUS class AUV", Proceedings of the MTS/IEEE Oceans'99 Conference, Seattle, USA, 1999.

[4] C. von Alt, B. Allen, T. Austin and R. Stokey, "Remote Environmental Measuring Units", Proceedings of the Autonomous Underwater Vehicle's 94 Conference, Cambridge, MA, USA, 1994.

[5] A. Gelb.. Applied Optimal Estimation, MIT Press, 1989.

[6] A. Matos, N. Cruz, A. Martins and F. L. Pereira, "Development and Implementation of a Low-Cost LBL Navigation System for an AUV", Proceedings of the MTS/IEEE Oceans'99 Conference, Seattle, USA, 1999.

[7] J. Silva, A. Martins and F. L. Pereira, "A Reconfigurable Mission Control System for Underwater Vehicles", Proceedings of the MTS/IEEE Oceans'99 Conference, Seattle, USA, 1999. 